Journal of Accident and Emergency Medicine 1995 12, 145-146
Correspondence: H. Rowley, Research Fellow in Otolaryngology, Department of Otolaryngology, Royal Liverpool University Hospital, P.O. Box 147, Liverpool L69 3BX, UK

\title{
Pharyngeal perforation: An easily missed finding following intra-oral injury
}

\author{
H.ROWLEY, J.CHRISTIAN \& A.DENNIS
}

Department of Otolaryngology, Our Lady's Hospital for Sick Children, Crumlin, Dublin.

\section{SUMMARY}

A 2-year-old child presented with pharyngeal perforation following a peroral injury with a toothbrush. Direct force applied to an object such as a toothbrush in the mouth may cause either superficial or penetrating injury within the oropharynx. A high index of suspicion is sometimes necessary to identify a pharyngeal perforation and if this is diagnosed we would advocate admission to hospital, restriction of oral intake, intravenous fluids and antibiotics, with close observation to ensure that healing occurs without development of further complications.

Key words: injury, perforation, pharynx

\section{INTRODUCTION}

The pharynx and oral cavity are particularly vulnerable to injury in children who fall with a toothbrush, pencil or other implement in the mouth. These injuries may go unrecognized until symptoms such as cervical pain and dysphagia and signs such as fever or subcutaneous emphysema appear. This case illustrates points regarding recognition and treatment of such injuries.

\section{CASE REPORT}

A 2-year-old female presented to the accident and emergency (A\&E) department $18 \mathrm{~h}$ after she had fallen with a toothbrush in her mouth. She sustained an intra-oral injury and vomited some blood stained vomitus at the time. By the following day she had ceased to eat or drink but had no other complaints. In particular she had no stridor or other airway problem.

On examination she had a mild pyrexia and on inspection of the oral cavity and pharynx, a small laceration on the posterior wall of the oropharynx was noted. There was no clinic evidence of surgical emphysema.
A lateral soft tissue radiograph of the neck showed air in the pre-vertebral space from $\mathrm{C} 2-\mathrm{C} 7$ with evidence of soft tissue swelling of the retropharyngeal space (Fig. 1). Initial haematological investigations revealed a raised white cell count of 18 with a leucocytosis. The child was admitted, given nil orally and was treated with intravenous fluids and systemic antibiotics; in this case augmentin (amoxycillin + clavulinic acid) was used.

Serial radiographs over the next 3 days showed a gradual clearing of air from the prevertebral space tissues (Fig. 2). By the fifth day, the child was eating and drinking normally and was allowed home. At

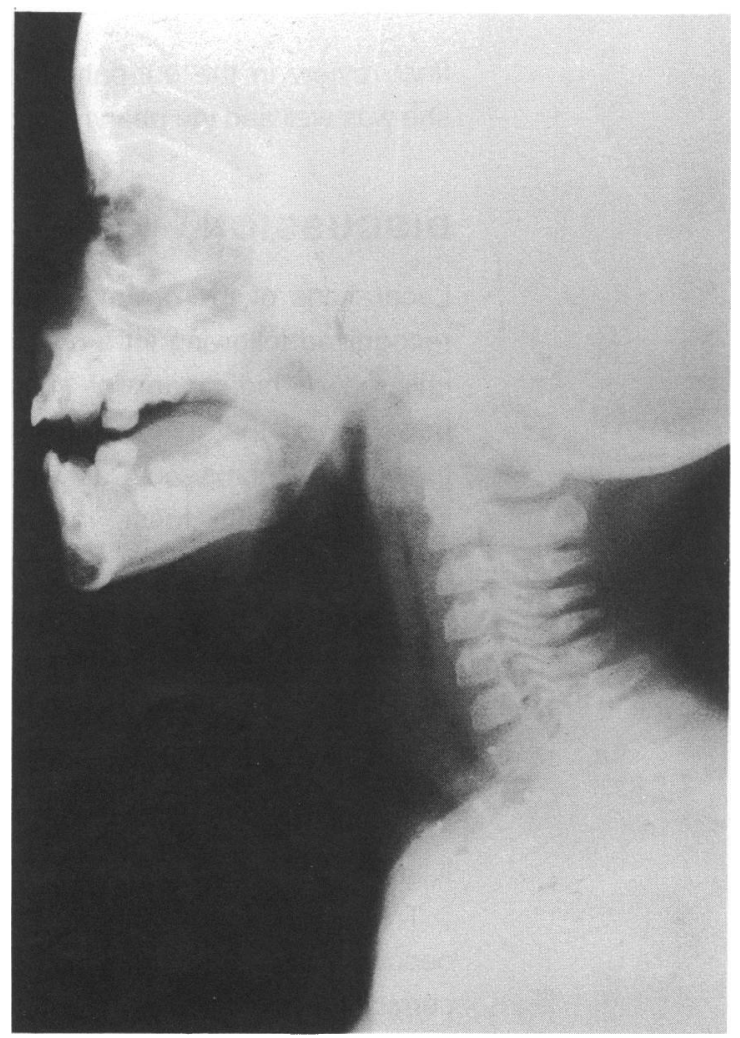

Fig. 1. Lateral soft tissue radiograph of neck showing presence of air in the pre-vertebral space. 


\section{H. Rowley et al.}

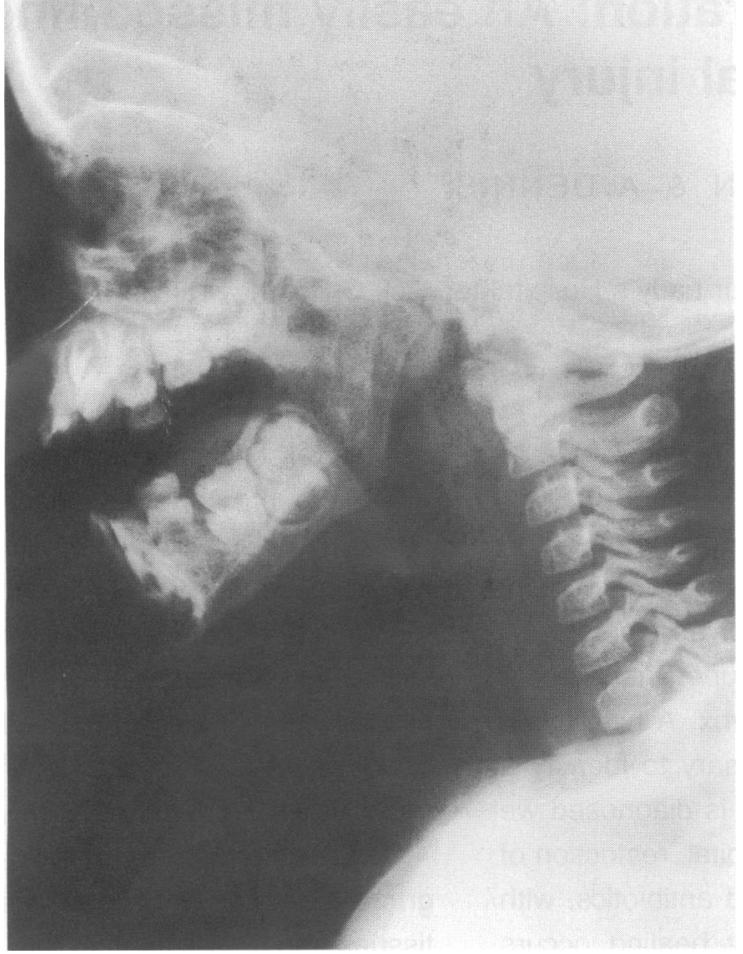

Fig. 2. Lateral soft tissue radiograph of neck showing some resolution of air from the prevertebral space compared with Fig. 1.

final review in the out-patient clinic 1 month later she was well and the pharynx had healed completely.

\section{DISCUSSION}

Lacerations of the oral cavity and palate are well recognized following intra-oral injury, particularly in children. A high index of suspicion is necessary however, so that injuries such as pharyngeal perforation are not missed. Patients often do not present immediately with these injuries but may do so up to $24 \mathrm{~h}$ later when the child ceases to eat or drink or indeed later still when the child becomes pyrexial and unwell. Clinical signs are quite subtle and, as in this case, all that one may see is a small, easily missed puncture wound or laceration of the pharyngeal wall. A radiograph may help as in this instance, when air may be seen in the pre-vertebral tissues.

Traditionally, perforation of the pharynx and oesophagus secondary to injury has been managed surgically. More recently non-operative medical management of traumatic pharyngo-oesophageal perforation in selected cases has been advocated. ${ }^{1}$ proved to be relatively safe, cost effective, resulting in no prolonged disability and a relatively short hospital stay.

Singer has proposed a management strategy for penetrating oropharyngeal injuries in which he advocates that the natural course for the majority of these injuries is spontaneous healing. ${ }^{2}$ It should be borne in mind however, that penetrating trauma to the parapharyngeal space may cause life threatening vascular injuries and penetration of the retropharyngeal space may lead to dissecting emphysema or mediastinitis. ${ }^{3}$

Not all patients have a uncomplicated recovery. Cornelio-Nieto et al. reported case of a 4-year-old girl who suffered a cerebral infarct secondary to an intra-oral injury from a spoon. ${ }^{4}$ Complications related to upper aerodigestive tract injuries carry a $10-15 \%$ mortality rate. ${ }^{5}$

It is important to heighten awareness of the general public that serious injuries may occur when children fall with such implements in the mouth. Likewise, it is important that medical staff who deal with such cases recognize that, while symptoms and signs may be subtle, such injuries may have serious consequences. When such an injury is suspected soft tissues radiographs should be performed. Once a perforation is diagnosed the patient must be admitted to hospital and a reasonable first line approach is that of close observation, restriction of oral intake, intravenous fluids and parenteral antibiotics.

We found serial radiographs to be helpful in demonstrating radiological clearance of air from the soft tissues. This may help monitor progress.

\section{REFERENCES}

1. Dolgin S.R., Kumar N.R., Wykoff T.W. \& Maniglia A.J. (1992) Conservative medical management of traumatic pharyngoesophageal perforations. Annals of Otology, Rhinology and Laryngology 101, 209-215.

2. Singer J.I. (1989) Management strategy for penetrating oropharyngeal injury. Paediatric Emergency Care 5, 250-252.

3. Fliss D., Goldstein Y. \& Tori F. (1988) Cervico mediastinal emphysema due to anterior oropharyngeal penetrating injury. Harefuah 114, 125-126.

4. Cornelio-Nieto J.O., Rodriguez-Murillo R.M., RamirezAguilera M.J., Lastra-Escudero L.G., Camejo-Reyes R.A. \& Medecigo-Castelain E. (1992) Cerebral infarct secondary to an intra-oral injury from a spoon. Boletin Medico Del Hospital Infantil de Mexico 49, 384-387.

5. Michel L., Grillo H.C. \& Malt R.A. (1981) Operative and nonoperative management of oesphageal perforations. Annuals of Surgery 194, 56-63. 